\title{
A Video Surveillance Oriented 3D Terrain Simplification Algorithm
}

\author{
X. Fu, J. L. Li, J. X. Zeng \\ School of Software, Nanchang Hangkong University \\ Jiangxi Nanchang, China
}

\begin{abstract}
In video surveillance fields, the effectiveness of 3D terrain description method is the base of improving monitoring effect, selecting positions of cameras to be deployed reasonably and scientifically. In this paper, a new local land surface roughness representation method is introduced, and then combined with features of camera forms the measurement of merging neighbouring regions. Experiments show the results of the proposed 3D terrain simplification method, and the effectiveness is also proved. For real 3D terrain data used in the experiments, the number of grids is reduced sharply. The difference between coverage rate to the source terrain and the simplification terrain is small, and has almost the same coverage rate when the number of cameras is enough, which means that covering the source terrain can be guaranteed by covering the simplification terrain.
\end{abstract}

Keywords-video surveillance; terrain simplification; terrain features; characteristics of camera; region merging

\section{INTRODUCTION}

In practical application of video surveillance, it is an important issue to realize effective monitoring to the whole interested region, where the contents needs researching include selecting the positions of cameras to be deployed, calculating whether each points in the region can be monitored, and achieving effective covering to the whole region with minimum number of cameras. Thus it can provide theory support and scientific reference to the deployment of camera nodes in practice, improve monitoring performance and save cost at the same time. For some complicated real 3D scene, description the terrain effectively is the base of achieving mentioned researches. It is found that traditional 3D terrain description adopted some discretization techniques include contour lines, triangulation and grid network. These methods focus on approximation to the real terrain surface with dense sampled points, which is different with the objective of video surveillance. In video surveillance fields, the most important objective is not the approximation to the real terrain, but the coverage by cameras. Dense sampled points will spend more computing time under this condition; moreover, excessive sampled points within flat regions will lead to calculating waste. In this paper, 3D terrain simplification algorithm oriented to video surveillance is researched.

The existed terrain simplification methods were all used to the dynamic generation of virtual environment, and some view point related methods were proposed to terrain simplification. Yang et al [1] presented three view-dependent criterions to reduce the complexity of terrain: view-frustum intersection, node orientation and screen space error. This method had higher speed in rendering virtual terrain scene. In [2], Du et al proposed multi-resolution model of terrain based on vision generating rule, and a method named remaining energy is introduced to reduce the area far from the viewer. A feature degree of a vertex is presented in [3] to indicate the valley or ridge feature for a vertex. Through calculating the feature degree of vertexes, the order of candidates of error metric could be changed. So the more important vertex could be preserved and more terrain features could be maintained. Han et al [4] used view-dependent local entropy based on surface roughness to simplify terrain near and Phong illumination model to simplify terrain far.

In the terrain simplification oriented to video surveillance, if cameras are regarded as view points, it is very similar with traditional terrain simplification used to terrain visualization. But they have following differences: Firstly, there is only one view point in terrain visualization, so regions far away from the view point can be simplified directly; while there are more than one view points in video surveillance, so regions far away from one point may close to others, they can not be simplified simply. Secondly, they have different objective. Terrain visualization focuses on visual quality, while video surveillance cares about the effectiveness of monitoring to interesting regions, that is, monitoring the real terrain can be guaranteed by covering the simplified terrain. What' s more, the invisible occlusion regions can be simplified in terrain visualization; while in video surveillance, the blind monitoring angle should be reduced by adjusting the position of view points (cameras) or increasing the number of camera. Finally, in terrain visualization, it has high requirement about rendering speed and needs real-time simplification; while in video surveillance, the terrain simplification is used to simulate and supply theory support for the deployment of camera in practice, the faster processing speed is the better, but not necessarily real time. In summary, traditional terrain simplification methods used to visualization can not be applied to video surveillance directly.

In this paper, terrain simplification method oriented to video surveillance was researched by combing the terrain features and the characteristics of camera. Firstly, local terrain surface roughness is calculated. Then, the intersection angle between neighbouring regions is used to compare with the field of view of camera. Whether the neighbouring regions can be merged or not is judged by both the surface roughness and the angle. Finally, the monitoring relationship between simplified terrain and source terrain is proved through experiments. 


\section{AlgORITHM OF TERRAIN SIMPLIFICATION}

In the application fields of video surveillance, many researchers use 2D rectangle to descript the target area needed to be monitored [5], at the same time, the source terrain data is often denoted by discretization rectangle grids [6]. So the terrain data before and after simplification are both indicated by rectangle in this paper.

\section{A. Simplification Based on Terrain Features}

In this paper, the local terrain roughness is used to represent the terrain feature. Traditionally, there are two methods to calculate the terrain roughness. The first one is shown in fig. 1(a), the roughness is expressed by the ratio between the area of real terrain region and that of the projection. The ratio equal to 1 means horizontal flat surface, and the ratio is smaller than 1 even if for inclined plane. This kind of calculation method is unsuitable to the application in this paper because the inclined plane with large tilt angle will has big terrain roughness and not be merged. The second traditional method is shown in fig. 1(b), the height difference between diagonal midpoints is treated as terrain roughness. This method is unsuitable to the application in this paper either, for example, as the terrain shown in fig. 1(c), the height difference between diagonal midpoints is small but the real terrain is very complicated.

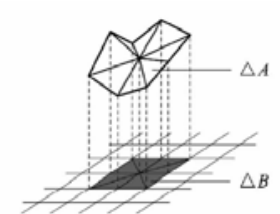

(a)

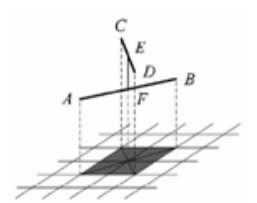

(b)

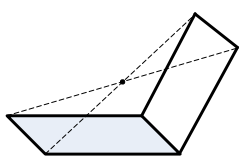

(c)
FIGURE I. TRADITIONAL TERRAIN ROUGHNESS DESCRIPTION METHODS. (A) THE RATIO BETWEEN THE AREA OF REAL TERRAIN REGION AND THAT OF THE PROJECTION; (B) THE HEIGHT DIFFERENCE BETWEEN DIAGONAL MIDPOINTS; (C) A FAILED

EXAMPLE FOR METHOD IN (B), THAT IS, THE HEIGHT DIFFERENCE BETWEEN DIAGONAL MIDPOINTS IS SMALL BUT THE REAL TERRAIN IS VERY COMPLICATED

Given two neighbouring rectangles $A B C D$ and $C D E F$ as shown in fig. 2, the rectangles may be the grids of source terrain data or new rectangles after being merged, and the local terrain roughness in this paper can be defined as:

$$
R=\{d(G), d(H)\}
$$

where $d(G)$ means the height difference between the midpoint $G$ of line $A E$ and its corresponding real terrain, $d(H)$ has similar meaning with $d(G)$ for midpoint $H$. When $R$ satisfies conditions in (2), further judgments will be done to see whether rectangles $A B C D$ and $C D E F$ can be merged into a new rectangle $A B F E$.

$$
\left\{\begin{array}{l}
|d(G)|<T_{1} \\
|d(H)|<T_{1} \\
d(G) \cdot d(H)>0
\end{array}\right.
$$

where $T_{1}$ is a threshold, $\|$ means computing the absolute value. In (2), the first two formulas indicate the terrain changes in local region are small. The third formula means $A E$ and $B F$ has the same inclining direction, thus prevention points $E$ and $F$ located at two sides of plane $A B C D$.

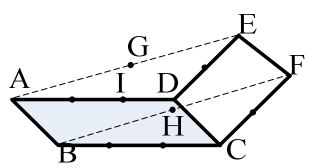

FIGURE II. NEW TERRAIN ROUGHNESS DEFINED BASED ON TWO NEIGHBOURING RECTANGLES

\section{B. Simplification Combined with Video Characteristics of Camera}

As mentioned above, the terrain simplification oriented to video surveillance has particularity and is different with traditional terrain simplification used to visualization, in this paper, we try to simplify 3D terrain by combining the video characteristics of camera. It is founded that beside visual distance, the main characteristics of camera included: field of view, that is, the visual region is located within an angle; orientation, only the side face to the camera is visible. fig. 3(a) and fig. 3(b) represent valley and ridge terrain respectively, $\vec{n}_{1}$ and $\vec{n}_{2}$ are the surface normal vectors of neighbouring regions. $\alpha$ is the intersection angle of two normal vectors, its value range is $\left[0^{\circ}, 180^{\circ}\right) \cdot \beta$ expresses the intersection angle between two neighbouring rectangles, its value range is $\left(0^{\circ}, 180^{\circ}\right]$. Obviously, there is $\beta=180^{\circ}-\alpha$, when $\alpha$ is $0^{\circ}$, then $\beta$ is $180^{\circ}$, that means the two neighbouring rectangles are coplanar; bigger $\alpha$ value corresponding to smaller $\beta$, which means narrower valley for valley terrain in fig. 3(a) and sharper ridge for ridge terrain in fig. 3(b).

If the angle meets the relationship in (3), and the terrain roughness satisfies (2), the rectangles $A B C D$ and $C D E F$ in fig. 2 can be merged into one new rectangle $A B F E$.

$$
\begin{cases}\alpha \leq \omega_{\vee} \cdot \theta & \text { valley terrain } \\ \alpha \leq \omega_{\wedge} \cdot \theta & \text { ridge terrain }\end{cases}
$$

where $\theta$ is the field of view of camera, $\omega_{v}$ is coefficient for valley terrain and $\omega_{\wedge}$ is coefficient for ridge terrain.

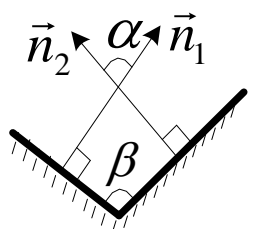

(a)

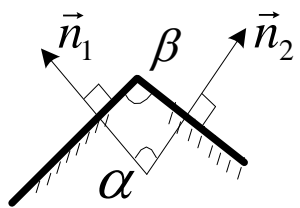

(b)
FIGURE III. INTERSECTION ANGLE BETWEEN TWO NEIGHBOURING RECTANGLES: (a) NORMAL VECTORS AND INTERSECTION ANGLE FOR VALLEY TERRAIN; (b) NORMAL VECTORS AND INTERSECTION ANGLE FOR RIDGE TERRAIN 


\section{EXPERIMENTAL RESULTS AND ANALYSIS}

\section{A. Simplification Results}

As shown in fig. 4(a), the source terrain data used in experiment is expressed in $65 \times 65$ uniform grids. There are 4225 rectangles totally. The field of view $\theta$ of camera is $60^{\circ}$. $\omega_{v}$ in (3) is set as 1.5 through experiment, which means the intersection angle between two neighbouring rectangles in valley terrain satisfying $\beta \geq 90^{\circ} . \omega_{\wedge}$ is set as 0.5 , which means $\beta \geq 150^{\circ}$ in ridge terrain. When $T_{1}$ in (2) is 1 , the simplified terrain is shown in fig. 4(b), there are 2251 rectangles; when $T_{1}$ is 1.5 , the results is shown in fig. 4(c), there are 888 rectangles remained. It can be seen from fig. 4 that the main terrain features are preserved after simplification, and the number of rectangles is reduced significantly, which is convenient for computation of next step.

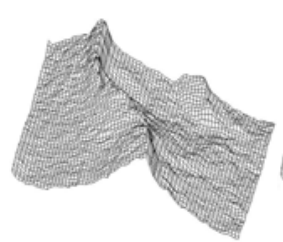

(a)

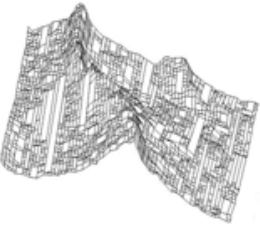

(b)

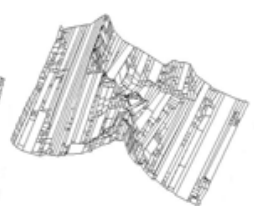

(c)
FIGURE IV. $\quad$ SIMPLIFICATION RESULTS. (a) Source Terrain; (b) Simplified Terrain When $T_{1=1}$; (c) Simplified Terrain When $T_{1=1.5}$.

\section{B. Effectiveness of the Simplification}

The effectiveness of the simplification is defined as when a point in the simplified terrain can be covered by a camera, the corresponding point in the real terrain can also be covered by the camera. For the whole interesting region, the effectiveness of terrain simplification means that the coverage ratio to the simplified terrain is approximate to the coverage ratio to real terrain. In the field of video surveillance, locating the cameras scientifically and covering the target area with the minimum number of camera is another research issue, which is out of the research scope of this paper, thus, we deploy cameras within the target area randomly and compare the coverage ratio before and after simplification.

The comparison of coverage ratio is given in fig. 5, coverage ratio curves under three different terrain conditions are drawn, the source terrain, the simplified terrain for $T_{1}$ is 1 and 1.5. It can be seen that the coverage ratios are upward trend with the increasing of the number of deployed cameras. The coverage ratios to the simplified terrain and to the source terrain are very close. When the number of cameras is more than 180 , there are similar coverage ratios to the simplified terrain and to the source terrain.

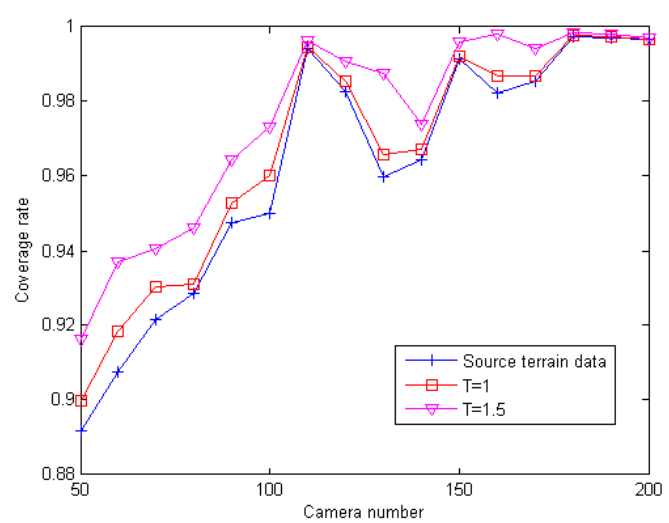

FIGURE V. COVERAGE RATIO CURVES UNDER THREE DIFFERENT TERRAIN CONDITIONS

\section{CONCLUSIONS}

In this paper, the differences between terrain simplification oriented to video surveillance and to dynamic virtual environment visualization are analyzed. Combined with terrain features and the video characteristics of camera, a terrain simplification oriented to video surveillance is proposed. Experiments show that the proposed method can reduce the number of terrain grid considerably, at the same time, it can keep the consistency of monitoring to the simplified terrain and the source terrain.

\section{ACKNOWLEDGEMENT}

This work is supported in part by the National Natural Science Foundation of China (Grant No. 61165011), the Science and Technology Project of Jiangxi Province (Grant No. 20122BBF60072) and the Youth Science Foundation of Jiangxi province, China (Grant No. 20132BAB211021).

\section{REFERENCES}

[1] Yang, C. Y., Zhang, J. X. \& Lin, Z. J., The real-time LOD algorithm for rendering the virtual terrain scene. Acta Geodaetica et Cartographica Sinica, 30(2), pp. 133-139, 2001.

[2] Du, J. L., Chi, Z. X. \& Du, W., Vision-based generating rule for real-time multi-resolution model. Journal of Image and Graphics, 8(11), pp. 12951298, 2003.

[3] Hua, H. Y. \& Zhao, H. C., Mesh simplification algorithm for terrain model based on feature degree. Journal of Computer-aided Design \& Computer Graphics, 23(4), pp. 594-599, 2011.

[4] Han, M., Chen, H. B. \& Zheng, D. C., Terrain simplification algorithm based on Morton compressing in block and hybrid generating rule. Journal of Computer-aided Design \& Computer Graphics, 26(2), pp. 293-301, 2014.

[5] Kong, L. H., Zhao, M. C., Liu, X. Y. \& Lu, J. L., Surface coverage in sensor networks. IEEE Transaction on Parallel and Distributed Systems, 25(1), pp. 234-243, 2014.

[6] Wen, J. G., Zhao, X. J., Liu, Q., Tang, Y. \& Dou, B. C., An improved land-surface albedo algorithm with DEM in rugged terrain. IEEE Geoscience and Remote Sensing Letters, 11(4), pp. 883-887, 2013. 